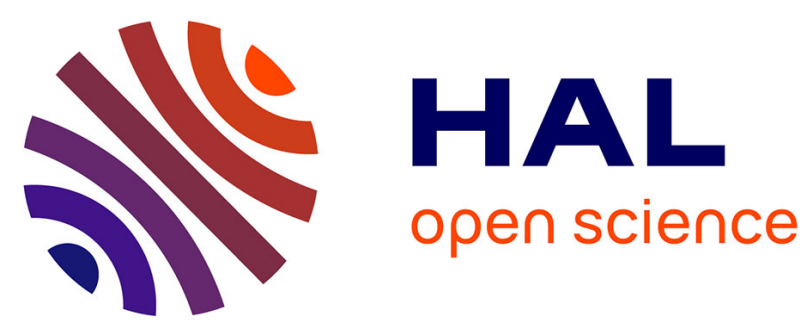

\title{
Offline Gain Adjustment with Constraints for Contour Error Reduction in High Speed Milling
}

Tan-Quang Duong, Pedro Rodriguez-Ayerbe, Sylvain Lavernhe, Christophe Tournier, Didier Dumur

\section{- To cite this version:}

Tan-Quang Duong, Pedro Rodriguez-Ayerbe, Sylvain Lavernhe, Christophe Tournier, Didier Dumur. Offline Gain Adjustment with Constraints for Contour Error Reduction in High Speed Milling. IEEE International Conference on Advanced Intelligent Mechatronics (AIM 2016), Jul 2016, Banff, Canada. 10.1109/aim.2016.7576767 . hal-01365977

\section{HAL Id: hal-01365977 \\ https://hal.science/hal-01365977}

Submitted on 13 Sep 2016

HAL is a multi-disciplinary open access archive for the deposit and dissemination of scientific research documents, whether they are published or not. The documents may come from teaching and research institutions in France or abroad, or from public or private research centers.
L'archive ouverte pluridisciplinaire HAL, est destinée au dépôt et à la diffusion de documents scientifiques de niveau recherche, publiés ou non, émanant des établissements d'enseignement et de recherche français ou étrangers, des laboratoires publics ou privés. 


\title{
Offline Gain Adjustment with Constraints for Contour Error Reduction in High Speed Milling*
}

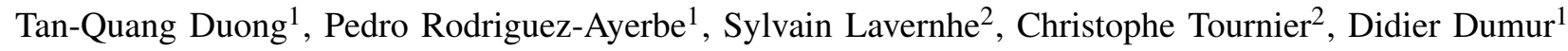

\begin{abstract}
In machining complex parts with high speed milling, small contour error (CE) and high surface quality are usually two major concerns. Many papers have focused on reducing $\mathrm{CE}$ by online contouring adaptation in the presence of disturbances and/or uncertain nonlinearities. Besides, calibration methods on pre-machining process such as smoothing the setpoints with optimized feedrate are also preferred. In contrast, less efforts have been done to analyze the relationship between profile geometry, control tuning or adjustment and effective $\mathrm{CE}$ in order to obtain $\mathrm{CE}$ as small as possible. To effectively deal with this challenge, an Offline Gain Adjustment (OGA) algorithm is developed in this paper. The simulated comparisons with existing fixed gain controller prove the efficiency of the proposed OGA method.
\end{abstract}

\section{INTRODUCTION}

Contouring control is one of the most crucial issues in industrial High Speed Machining (HSM) process to ensure precision and quality of the produced parts. Its objective is to obtain small CE which is defined by the shortest distance between the actual position and the desired contour. In the ideal case, zero tracking error leads to zero CE. However, it cannot be obtained due to critical response time, friction or disturbances, etc. Furthermore, decreasing tracking error sometimes unfortunately increases the CE [1]. On one hand, the industrial HSM confronts with different geometries on the complex parts, in which the tolerance may be different all over the part to be machined. On the other hand, the machine usually uses a controller with predefined fixed gains, such as proportional or feed forward controller, to fulfill its job. It is clear that the traditional controllers limit the contour performance on the whole machined part. Consequently, adaptation efforts during machining or pre-machining process, in other words an online or offline contouring adaptation respectively, could be a useful remedy for these challenges.

In online contouring adaptation, authors usually propose an approximated $\mathrm{CE}$ model and use a specific adaptive control technique to minimize the $\mathrm{CE}$, with or without the presence of disturbances or nonlinearities. It has been stated that in order to reduce the CE more, the axes should

\footnotetext{
*This work, $\mathrm{N}^{0} 2014-812 \mathrm{D}$ - Projet OMEGA, is supported by the DIGITEO foundation

${ }^{1}$ Tan-Quang Duong, Pedro Rodriguez-Ayerbe and Didier Dumur are with L2S, CentraleSupélec - CNRS - Univ. Paris-Sud, Université Paris-Saclay, 91190 Gif-sur-Yvette, France tanquang.duong@centralesupelec.fr; pedro.rodriguez@centralesupelec.fr;

didier.dumurecentralesupelec.fr

${ }^{2}$ Sylvain Lavernhe and Christophe Tournier are with LURPA, ENS Cachan, Univ. Paris-Sud, Université Paris-Saclay, 94235 Cachan, France sylvain.lavernhedens-cachan.fr; christophe.tournier@ens-cachan.fr
}

be coordinated [1], [2], [3]. Koren et al. [1] proposed a variable-gain Cross Couple Controller (CCC), in which the $\mathrm{CE}$ is estimated by a function of feedback tracking error, multiplied by appropriate gains to generate different extra control efforts. The CE model can also be estimated in task space. Chiu and Tomizuka [4] proposed a task coordinated frame attached locally to the desired contour. By this way, some projections of the actual position tracking error in this moving task coordinate frame can be used to approximate the $\mathrm{CE}$ for feedback controller design [3]. More accurate global task coordinate frame (GTCF) has been developed by B. Yao et al. [2]. It calculates the CE exactly to its first order approximation. Concerning adaptive control techniques, Yan et al. [5] proposed a self tuning adaptive control strategy for reducing tracking error while the CCC structure was used for degrading the CE. In addition, Uchiyama et al. [6] developed a robust adaptive controller that has a realtime estimator of the upper bounds of unknown parameter and disturbance magnitude, yielding significant improvement in contour performance. Recently, David et al. [3] used an Adaptive Robust Control (ARC) approach, which has been proposed by B. Yao [7], to efficiently deal with tool deflection effect in the aim of $\mathrm{CE}$ reduction.

The offline contouring adaptation usually refers to precalibrated error compensation. This method uses the error information of each operation to alter or calibrate the process in the subsequent operations [8]. Habibi et al. [9] used a modified path in the purpose of improving contour performance while the tool deflection estimation and geometric error analysis are considered. Jaen-Cuellar et al. [10] proposed a new methodology for PID control tuning by coupling the gain phase margin method with the genetic algorithm, providing a good response in servo system, which is used under the discrete mathematical model. However, this gain tuning method considers tracking performance rather than contour performance. To the best of the authors' knowledge, there are few works on generating offline a set of variable gains in order to reduce the $\mathrm{CE}$ during the machining process.

Thanks to the above syntheses, this study aims at proposing an OGA algorithm, taking into account the axes coordination and the effects of profile's geometry on CE behaviors, in yielding high contour performance.

The rest of paper is organized as follows: Section II is dedicated to the problem formulation, while the detailed explanation of OGA method is presented in Section III. Simulation results are then discussed in Section IV. Finally, the paper is concluded in Section V. 


\section{PROBLEM FORMULATION}

In Fig. 1.a, classical definitions of biaxial contouring control with fixed gain controllers, in XZ plane for example, are illustrated. At $k^{\text {th }}$ instant, $P_{d, k}$ and $P_{a, k}$ are the desired and actual positions of the tool path respectively; $E_{X, k}$ and $E_{Z, k}$ are namely the tracking errors of $\mathrm{X}$ and $\mathrm{Z}$ axes while $E_{k}$ and $\varepsilon_{k}$ are the norm of the tracking error and the CE respectively; $K_{X}$ and $K_{Z}$ are the fixed control gains for position loop of $\mathrm{X}$ and $\mathrm{Z}$ axes. The gain direction is defined as same as the direction of axis tracking error to facilitate the analysis. The desired contour represents the continuous trajectory to be followed by the axes associated to the setpoints generated by tool path interpolation. Meanwhile, the actual contour usually differs from the desired one by the CE.

The fact is that geometry changing along the desired profile combined with the inherent tracking error is one of the major factors causing oscillations of the $\mathrm{CE}$ around the desired contour. For the purpose of $\mathrm{CE}$ reduction without considering the tracking error, there is a need to analyze the relationship between the future displacement of the axes and the present relative position of cutting tool compared with the desired contour.

- In the case of Fig. 1.b, the CE $\varepsilon_{k+1}$ could be smaller than the previous one $\varepsilon_{k}$ if the cutting displacement on the $\mathrm{X}$ axis is greater than that on the $\mathrm{Z}$ axis. This could be obtained if $K_{X, k}$ is greater than $K_{Z, k}$.

- Conversely, in the case of Fig. 1.c, the CE reduction can be obtained if the cutting displacement on the $\mathrm{Z}$ axis is greater than that on the $\mathrm{X}$ axis. It means that $K_{Z, k}$ should be greater than $K_{X, k}$.

Therefore, a suitable change of control gain should be derived from the relative position of cutting tool compared with the desired contour.

However, the variable gain cannot have infinite values, but should be bounded in an admissible range to maintain the kinematic limits of the machine's axes, including the velocity, acceleration and especially jerk and also to guarantee appropriate stability performances of the controlled loops. Not only the amplitude but also the variation frequency of these variable gains should be taken into account. This is due to the fact that servo system is usually controlled by a

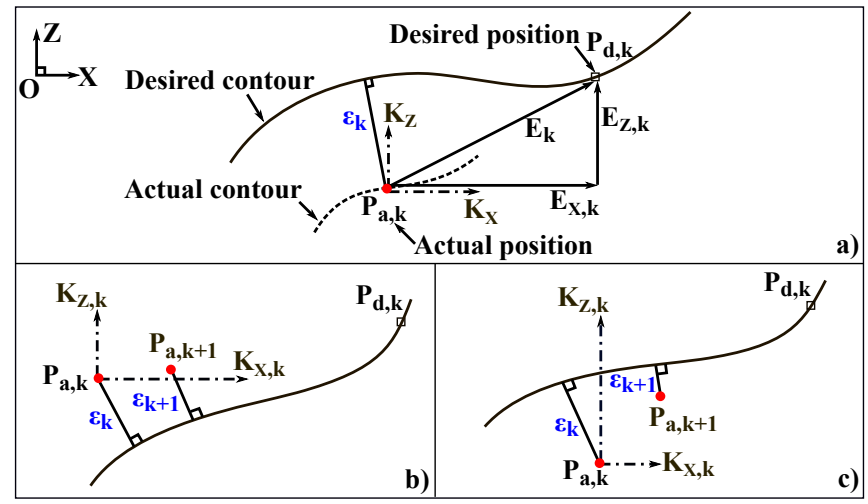

Fig. 1. Biaxial contouring control cascaded structure, with position, velocity and current loop ranging from the outer to inner loop respectively. Therefore, oscillation in the outer loop may be harmful to the inner one.

Another important issue is to determine the criterion that allows to generate good ratio of gains that can reach the objective and respect the constraints. There are two methods to address this concern, namely the single evaluation and the mean evaluation of the $\mathrm{CE}$.

- The former is proposed in Fig. 2.a where the gains $K_{X, k}$ and $K_{Z, k}$ are chosen so that $\varepsilon_{k+1}$ is smaller than $\varepsilon_{k}$. Then, the same idea is applied on the next instants.

- The latter is shown in Fig. 2.b which refers to a concept of receding window. The profile is first divided into contiguous segments, called windows $w_{i}$. The OGA algorithm is applied in one such window to find out the specific set of discrete gains so that the mean square of $\mathrm{CE}, \mathrm{MSE}(\mathrm{CE})$, is minimum. The window is afterwards receded to the next one and the algorithm is repeated.

One can note that both methods are capable of reducing the CE. However, with the first method, the actual position $P_{a, k}$ will easily cross over the profile and generate a non null CE. It can create oscillations on the control signal and on the resulting geometry. Meanwhile, the second one allows to consider the geometric characteristic of the desired contour as well as to generate a smooth control signal. Hence, the latter is preferred and developed in the following sections.

\section{OFFLINE GAIN ADJUSTMENT METHOD}

Building on the above ideas, the offline calculation method consists of two main steps:

- Implement the OGA algorithm to generate the selected variable gains.

- Verify these gains with axis behaviors through a nonlinear axis model.

Firstly, the OGA algorithm is illustrated in Fig. 3, where the inputs are the axis setpoints and the initial configurations, including admissible gain range, gain step, window length and predefined gain function, while the only output is a set of selected variable gains. In addition, the working mechanism is based on receding window without overlap.

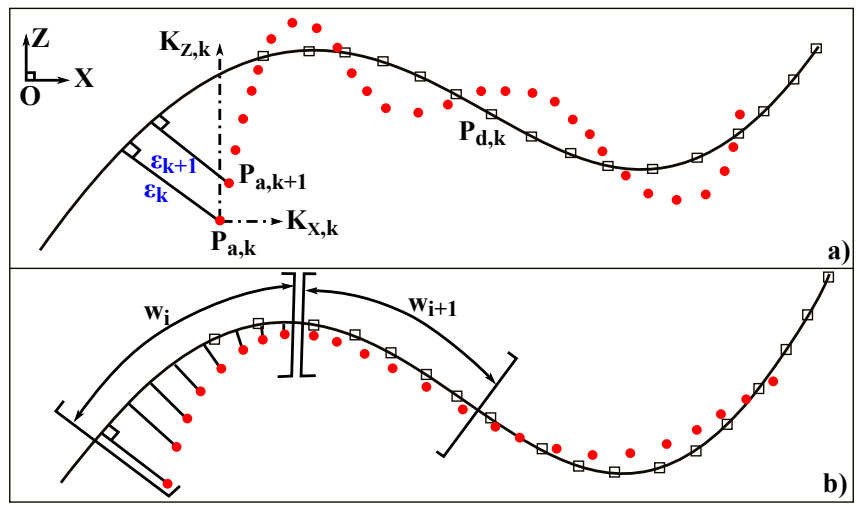

Fig. 2. CE evaluation 
In each window, the OGA is performed through four major tasks, namely:

- Task 1 requires that the initial configurations and the selected gains of previous window serve as the inputs. Firstly, the gain amplitude is tuned by a gain step within the admissible gain range. Then each value of gain amplitude is used in the predefined gain function to generate a set of discrete gains.

- Task 2 calculates the simulated positions from the first instant of trajectory until the last instant of the present window based on the nonlinear axis model. The inputs are the setpoints and the set of discrete gains produced by Task 1 .

- Task 3 aims at calculating the $\mathrm{CE}$ of present window through a CE model, in which the simulated positions from Task 2 and the setpoints are the inputs.

- Task 4 refers to the choice of the best set of discrete gains for the axes so that the $\operatorname{MSE}(\mathrm{CE})$ generated by Task 3 is minimum. The output of this task is a set of selected variable gains.

Afterwards, the selected variable gains are saved and updated for latter use. The window is then shifted in a receding manner and the algorithm is repeated until the last window. Eventually, the final set of the selected variable gains is obtained.

The verification step is then performed as in Fig. 4, where the whole gain produced by the OGA is applied to the nonlinear axis model. If the simulated responses have high contour performance and satisfy both the kinematic constraints of the machine's axes and the current limitation,

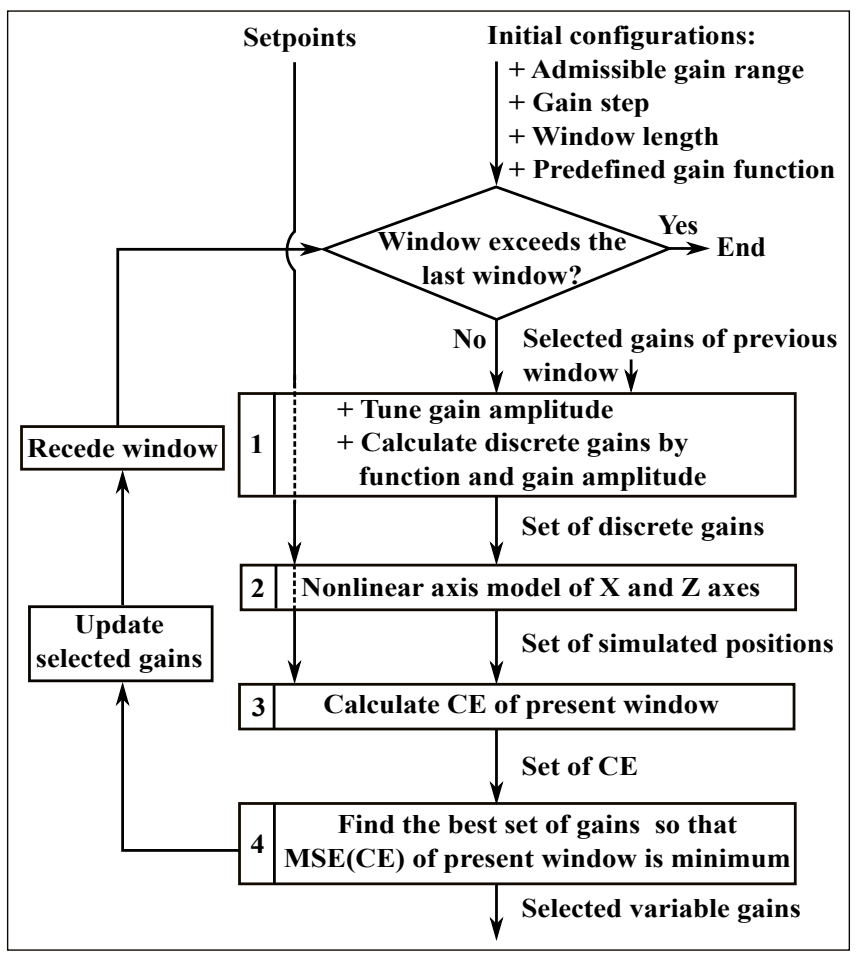

Fig. 3. Offline Gain Adjustment (OGA) algorithm these gains are used in the real machine tool to generate the actual positions of the axes with minimized CE. If it is not verified, some modifications such as modifying window length or finding other admissible gain ranges must be done to produce a better set of variable gains.

The kinematic constraints, setpoints generation, CE model, nonlinear axis model, admissible gain range, window length and predefined gain function are explained in detail as follows.

\section{A. Kinematic Constraints and Setpoints Generation}

Biaxial contouring control of the 5-axis MIKRON UCP 710 machining center (Fig. 4 bottom) is considered as the case study in this paper, in which the velocity, acceleration and jerk constraints of the concerned axes are given in Table I.

Generally speaking, setpoints generation in the Computer Numerical Control (CNC) is one of the major tasks in which the input is a reference tool path with a programmed feedrate and the output is a sequence of axis setpoints which has to produce a smooth movement, respecting the kinematic constraints. To effectively deal with this scheme, the VPOp algorithm which has been developed in [11] is used. This solution can be applied to any articulated mechanical structure.

TABLE I

KINEMATIC CHARACTERISTICS OF MIKRON MACHINE

\begin{tabular}{|c|c|c|c|}
\hline & $V_{\max }(\mathrm{m} / \min )$ & $A_{\max }\left(\mathrm{m} / \mathrm{s}^{2}\right)$ & $J_{\max }\left(\mathrm{m} / \mathrm{s}^{3}\right)$ \\
\hline $\mathrm{X}$ axis & 30 & 2.5 & 10 \\
\hline $\mathrm{Z}$ axis & 30 & 2.1 & 100 \\
\hline
\end{tabular}

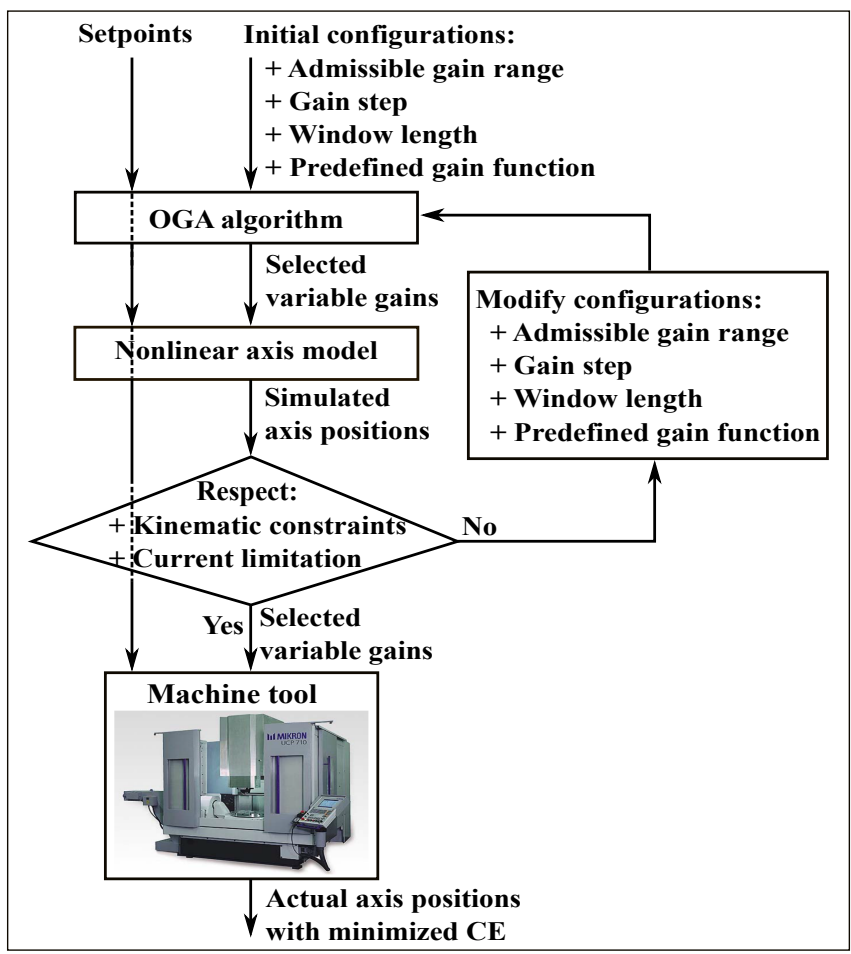

Fig. 4. Full offline calculation method of variable gains 


\section{B. Contour Error Model}

In the context of offline calculation, the simulated CE can be calculated at each instant using the line interpolation method [12]. Moreover, the sign of the $\mathrm{CE}$ is proposed to facilitate the analysis. In Fig. 5, the $\mathrm{CE} \varepsilon_{k}$ is computed by the following three steps:

- Step 1: At the $k^{\text {th }}$ instant of the simulated machining, calculate the tracking errors $E_{i}$, with $i=1: k$.

- Step 2: Find the minimum tracking error, $E_{i}$ for example, corresponding to the points $P_{a, k}$ and $P_{d, i}$.

- Step 3: Find the CE, which is the shortest distance from the actual position $P_{a, k}$ to the previous and next segments of the point $P_{d, i}$, yielding

$$
\varepsilon_{k}=\frac{\left|\overrightarrow{P_{d, i} P_{d, i+1}} \times \overrightarrow{P_{d, i} P_{a, k}}\right|}{\left|\overrightarrow{P_{d, i} P_{d, i+1}}\right|}
$$

The sign of $\varepsilon_{k}$ is defined by the sign of the scalar dot product of normal vector at point $P_{d, i}, \overrightarrow{n_{i}}$, and the tracking error vector $\vec{E}_{i}$. Therefore,

$$
\operatorname{sign}\left(\varepsilon_{k}\right)=\operatorname{sign}\left(\overrightarrow{n_{i}} \cdot \overrightarrow{E_{i}}\right)
$$

\section{Nonlinear Axis Model}

As seen in Fig. 6, a classical cascaded control structure, of $\mathrm{X}$ axis for example, is presented. In an external position loop, a proportional controller with a feed forward (FFW) action is considered while it is internally controlled by current and speed PI controllers of velocity loop [13]. From this framework, $X_{d}, X_{a}, V_{X d}$ and $E_{X}=X_{d}-X_{a}$ are desired position, actual position, desired velocity and tracking error of $X$ axis respectively, while $K_{c}, K P_{X}[\mathrm{~m} / \mathrm{min} / \mathrm{mm}]$ and $K F_{X}$ are namely conversion factor from $\mathrm{mm} / \mathrm{s}$ to $\mathrm{m} / \mathrm{min}$, proportional gain and FFW gain. Furthermore, the nonlinear characteristics of the axis due to frictions developed in [14] are used for reproducing the nonlinear effects of the machine axes.

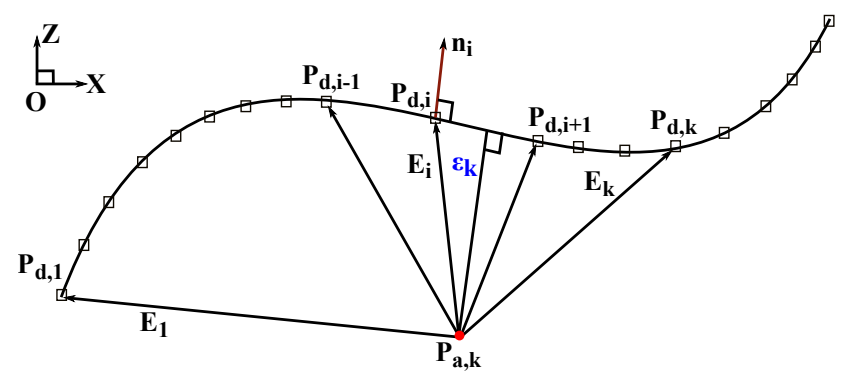

Fig. 5. Contour error model

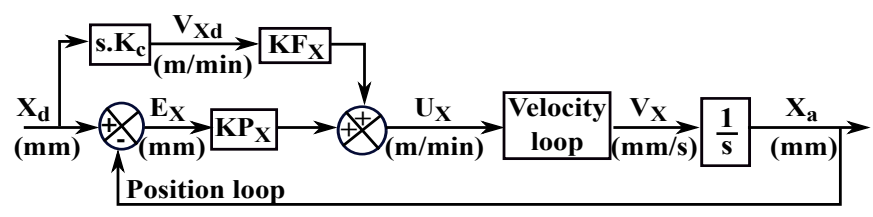

Fig. 6. FFW control structure

\section{Admissible Gain Range}

According to the control structure in Fig. 6, the tracking following is the main purpose of each axis. Given $K P$ and $K F$ are two control gains to tune in the range of $[1: 0.5$ : $9](\mathrm{m} / \mathrm{min} / \mathrm{mm})$ and $[0: 0.1: 1.2]$ respectively. One value of $K P$ combined with one value of $K F$ forms one control configuration of this kind of controller.

Respecting both the kinematic constraints and the current limitation of servo drive, there are namely 72 (green circles) and 108 (red squares) admissible gain configurations for $\mathrm{X}$ and $\mathrm{Z}$ axes, as given in Fig. 7. It is clear that the higher value of $K P$, the smaller value of $K F$ and vice-versa.

In fact, contour following of the desired profile in Fig. 8.a is mainly concerned. Therefore, there are $72 \times 108$ possibilities to perform such a control, in which the best choice with the minimum $\operatorname{MSE}(\mathrm{CE})$ is $K P=6[\mathrm{~m} / \mathrm{min} / \mathrm{mm}]$ and $K F=0.1$ for both axes.

\section{E. Window Length and Predefined Gain Function}

From the analysis in Section II, changing the geometry along the tool path usually changes the sign of CE. Thus, the window lengths are proposed to depend on the geometric characteristics all over the profile, as shown in Fig. 8. Each of three consecutive setpoints can compute one curvature value which may be positive, negative or zero for convex curve, concave curve or straight line respectively. The proposed idea is to detect each window having the same curvature characteristic as well as reflecting the transient part between two kinds of curvature.

The predefined gain function is one of the most important factors of the OGA algorithm. In Fig. 9.a, if the window $w_{i}$
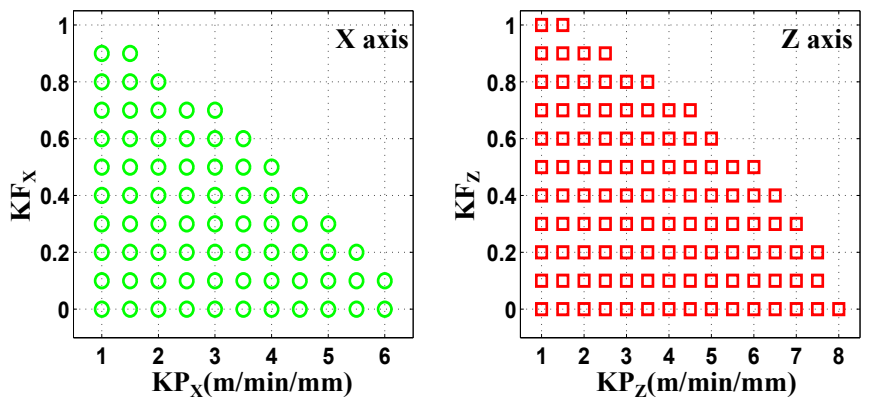

Fig. 7. Admissible gains with $J X_{\max }=10\left(\mathrm{~m} / \mathrm{s}^{3}\right)$ and $J Z_{\max }=100\left(\mathrm{~m} / \mathrm{s}^{3}\right)$

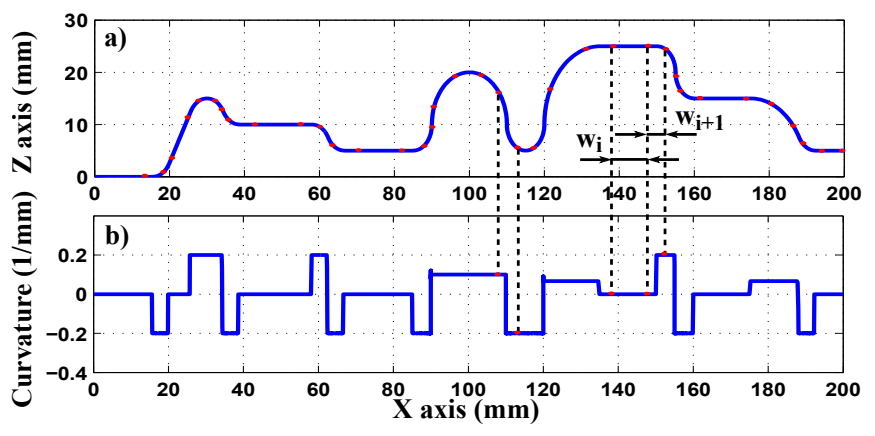

Fig. 8. Window length in function of curvature 
has the length $N_{i}$, the gain function must satisfy the following requirements:

- Calculate $N_{i}$ discrete gains in such a window.

- Allow to tune its amplitude inside the admissible gain range and to manage its variation frequency.

There are many approaches to formulate this kind of gain function such as using high order curve equation, etc. In this paper, the proposed function consists of a sinusoidal part and a linear part, formed by (3) and (4), respectively.

$$
\begin{aligned}
& K_{k: k+p_{i}-1}=-A_{i} \sin \left(2 \pi f_{i} . t\right)+A_{i}+K_{k-1} \\
& K_{k+p_{i}: k+N_{i}-1}=K_{k+p_{i}-1}
\end{aligned}
$$

where $p_{i}, A_{i}=\left[K_{\min }-K_{k-1}: \Delta_{K}: K_{\max }-K_{k-1}\right] / 2, t=$ $\left(p_{i} T_{e}\right) \times\left[1 / 2: 1 /\left(p_{i}-1\right): 3 / 2\right]$ and $f_{i}=1 /\left(2 p_{i} T_{e}\right)$ are the length, gain amplitude array, time array and variation frequency of the sinusoidal part, in which $K_{\min }$ and $K_{\max }$ are namely the lower and upper bounds of the admissible gain range, $T_{e}$ is the sampling time and $\Delta_{K}$ is the gain step. The smaller value of $\Delta_{K}$, the more case of gain amplitude tuning, and will result in longer computation time

As seen in (3), the first value of time array produces the first gain in the window, $K_{k}=K_{k-1}$. This means that the first discrete gain in each window is equal to the last gain of the previous one. Besides, (4) mentions that all discrete gains in linear part are equal to the last gain of the sinusoidal part. Therefore, for a specific window, the parameters to tune are $A_{i}$ and $p_{i}$ or $f_{i}$.

Finally, the desired behavior of variable gains is expected as same as Fig. 9.b.

\section{RESULTS}

Fig. 10.a firstly describes the result of setpoints generation by VPOp algorithm, with desired program feedrate of
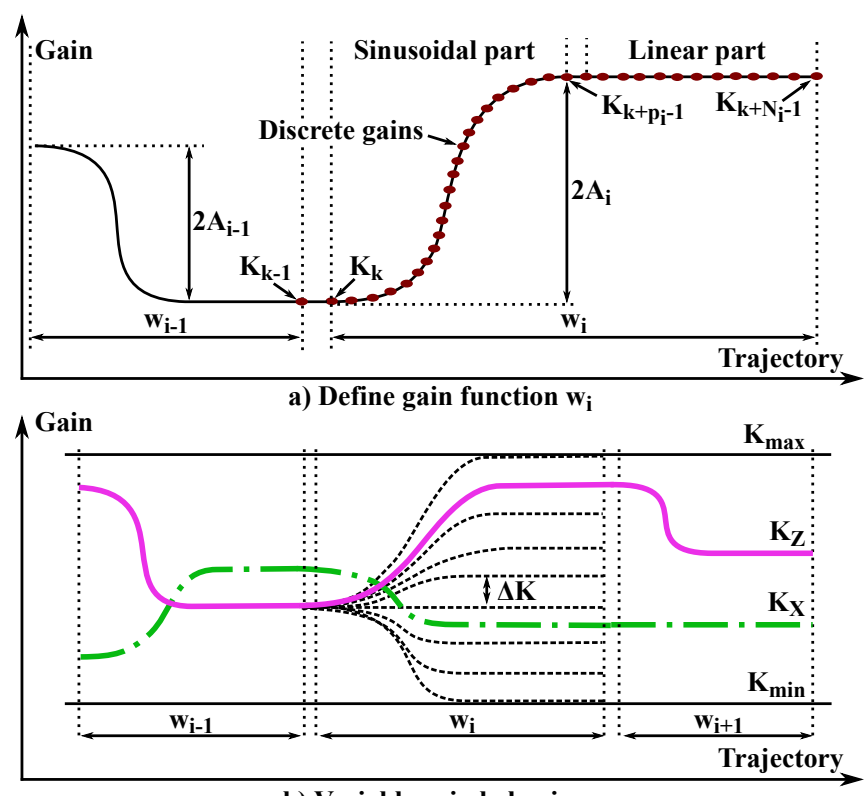

b) Variable gain behaviors

Fig. 9. Predefined gain function $3(\mathrm{~m} / \mathrm{min})$, interpolation tolerance of $20 \mu \mathrm{m}$ and the sampling time $T_{e}=1 \mathrm{~ms}$.

Two cases of study based on the control structure in Fig. 6 are conducted to highlight the efficiency of the proposed control method compared with the traditional one, namely:

- Fixed $K P$ and fixed $K F$. (C1)

- Adjusted $K P$ with OGA algorithm and fixed $K F$. (C2)

The best fixed gains and the admissible gain range for $\mathrm{C} 1$ and $\mathrm{C} 2$ respectively are chosen in Table II, starting from the analysis on Section III.D. The gain step and variation frequency of the sinusoidal part are also tuned to obtain the high contouring performance satisfying the constraints. This scheme leads to the choices of $\Delta_{K}=0.03(\mathrm{~m} / \mathrm{min} / \mathrm{mm})$ and $f_{i}=10 \mathrm{~Hz}$, which means $p_{i}=1 /\left(2 f_{i} T_{e}\right)=50$.

Although the OGA allows the adjustment on control gains of both axes, as a result of kinematic constraints, especially the jerk limitation, only the $K P_{Z}$ is adjusted while the $K P_{X}$ is kept fixed, as seen from Fig. 10.b. The sign changes of the $\mathrm{CE}$ in Fig. 10.c illustrate that the actual contour has oscillations around the desired one, with the maximum $\mathrm{CE}$ amplitude under $3.5 \mu \mathrm{m}$. In Fig. 10.d, the CE of C2 has a centered normal distribution, mainly ranging from -0.3 to $0.3 \mu \mathrm{m}$ while that in the case of $\mathrm{C} 1$ with the best fixed

TABLE II

CONTROL GAINS AND CONTOUR PERFormance

\begin{tabular}{|l|l|l|}
\hline & C1 & C2 \\
\hline$K P_{X}(\mathrm{~m} / \mathrm{min} / \mathrm{mm})$ & 6 & {$[5: 6]$} \\
\hline$K F_{X}$ & 0.1 & 0.1 \\
\hline$K P_{Z}(\mathrm{~m} / \mathrm{min} / \mathrm{mm})$ & 6 & {$[5.5: 6.5]$} \\
\hline$K F_{Z}$ & 0.1 & 0.1 \\
\hline \hline$M S E(\mathrm{CE})\left(\mu \mathrm{m}^{2}\right)$ & $\mathbf{0 . 7 6}$ & $\mathbf{0 . 2 5}$ \\
\hline Improvement & Reference & $\mathbf{6 7 . 1 1} \%$ \\
\hline
\end{tabular}
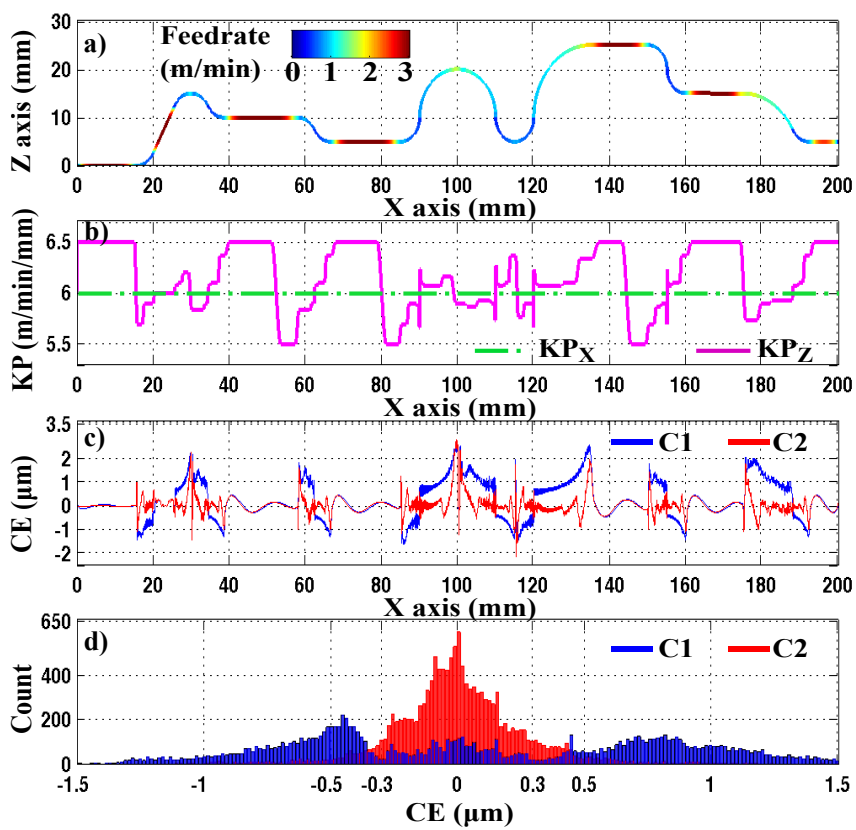

Fig. 10. Ajusted gains by OGA and contour performance 
gains ranges from 0.3 to $1.5 \mu \mathrm{m}$ for both sides of the desired contour. From Table II, it is clear that the MSE(CE) of C2 is reduced by about two thirds compared with that of the traditional case.

Fig. 11 shows that the feedrate and the axis jerk are quite the same for both cases, respecting the kinematic constraints shown in Table I. Meanwhile, the comparison of reference current signals issued from the velocity controller and applied to the current loop of $\mathrm{Z}$ axis for both cases is illustrated in Fig. 12, in which their maximum relative difference is around 3\%. It can be said that the variable control gains of $\mathrm{Z}$ axis does not deteriorate the control current signal.

\section{CONCLUSIONS}

In the effort of reducing the $\mathrm{CE}$ without considering the axis tracking errors, it can be concluded that geometry evaluation between the effective $\mathrm{CE}$ and the desired profile is a valuable clue. It allows to produce a good ratio of control gains on the axes. Consequently, an OGA algorithm has been developed through a systematic process to achieve the
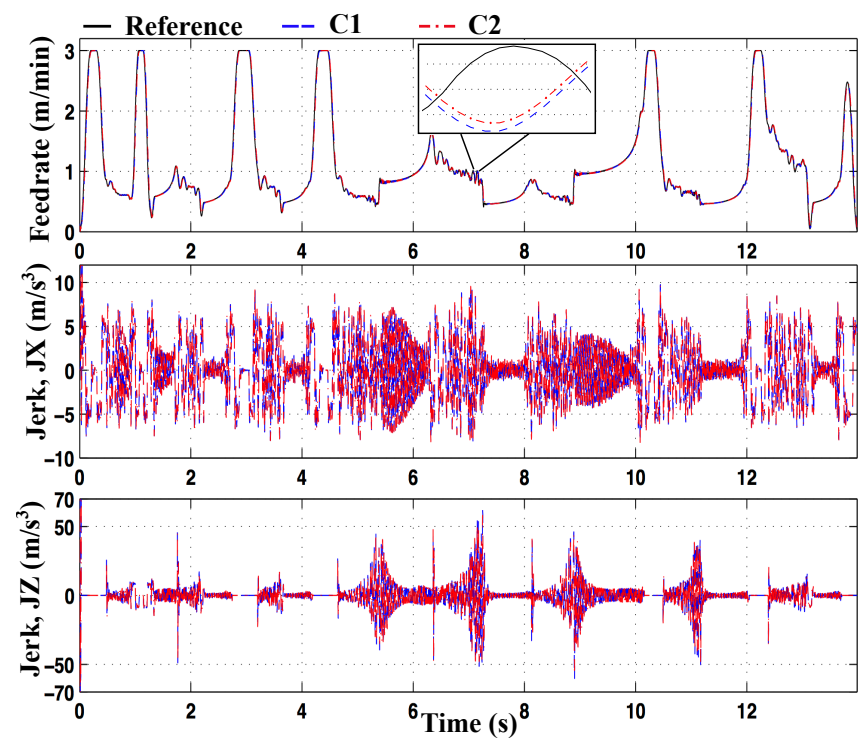

Fig. 11. Feedrate and axis jerk

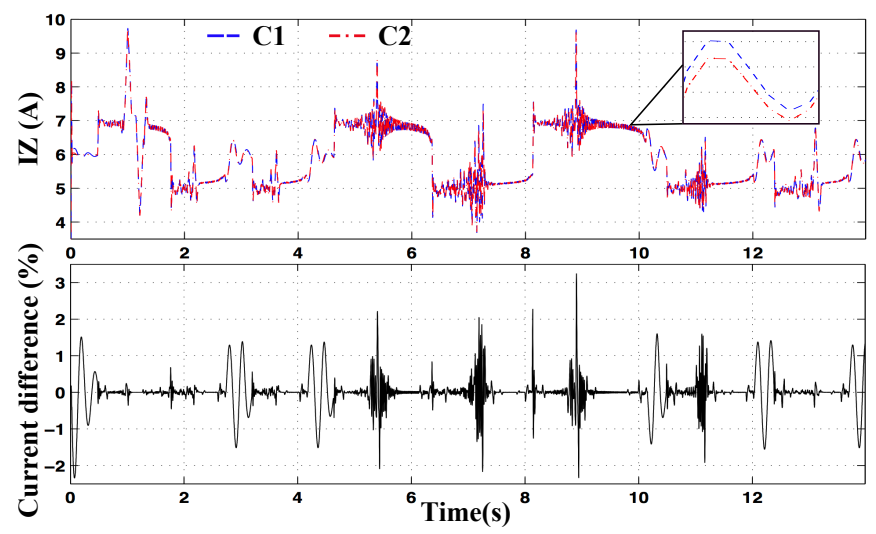

Fig. 12. Control current of $\mathrm{Z}$ axis purpose. The optimizing capacity of the proposed strategy depends on the value of gain step and other initial configurations, including admissible gain range, window length and predefined gain function. In particular, defining window length by the function of curvature is a good access to the geometry evaluation of the desired profile. It is also interesting to note that the gain function developed allows the control gains to be flexible in changing its amplitude inside the admissible gain range and to easily manage its variation frequency. Finally, the effectiveness of OGA has been validated by comparison with the traditional fixed gain controller, showing that not only the $\mathrm{CE}$ is significantly reduced but also the kinematic constraints of the machine's axes and the control current remain satisfied. Other methods to formulate the gain functions and their experimental tests will be a part of future works.

\section{REFERENCES}

[1] Y. Koren and C.-C. Lo, "Variable-gain cross-coupling controller for contouring," CIRP Annals - Manuf. Techno., vol. 40, no. 1, pp. 371374, January 1991.

[2] B. Yao, C. Hu, and Q. Wang, "An orthogonal global task coordinate frame for contouring control of biaxial systems," Mechatronics, IEEE/ASME Transactions, vol. 17, no. 4, pp. 622-634, August 2012.

[3] T. A. Davis, Y. Shin, and B. Yao, "Adaptive robust contour of circular machining contour error using global task coordinate frame," ASME. J. Manuf. Sci. Eng., vol. 137, no. 1, p. 8, February 2015.

[4] G.-C. Chiu and M. Tomizuka, "Contouring control of machine tool feed drive systems: a task coordinate frame approach," Control Systems Technology, IEEE Transactions, vol. 9, no. 1, pp. 130-139, January 2001.

[5] M.-T. Yan, M.-H. Lee, and P.-L. Yen, "Theory and application of a combined self-tuning adaptive control and cross-coupling control in a retrofit milling machine," Mechatronics, vol. 15, no. 2, pp. 193-211, March 2005.

[6] N. Uchiyama and S. Sano, "Robust adaptive contouring control for biaxial feed drive systems," Int. J. of Mach. Tools and Manuf., vol. 49, pp. 1204-1213, June 2009.

[7] B. Yao, "High performance adaptive robust control of nonlinear systems: a general framework and new schemes," Decis. and Control, Proce. of the 36th IEEE Conf., vol. 3, pp. 2489-2494, December 1997.

[8] P. D. Ngo and Y. C. Shin, "Milling contour error control using multilevel fuzzy controller," Int. J. Adv. Manuf. Technol., vol. 66, no. 9-12, pp. 1641-1655, August 2013.

[9] M. Habibi, B. Arezoo, and M. Nojedeh, "Tool deflection and geometrical error compensation by tool path modification," Int. J. of Mach. Tools and Manuf., vol. 51, no. 6, pp. 439-449, June 2011.

[10] A. Y. Jaen-Cuellar, R. de J. Romero-Troncoso, L. Morales-Velazquez, and R. A. Osornio-Rios, "Pid-controller tuning optimization with genetic algorithms in servo systems," Int. J. of Advan. Robot. Syst., vol. 10, no. 324, p. 14, September 2013.

[11] X. Beudaert, S. Lavernhe, and C. Tournier, "Feedrate interpolation with axis jerk constraints on 5-axis nurbs and g1 tool path," Int. J. of Mach. Tools and Manuf., vol. 57, pp. 73-82, June 2012.

[12] G. Zhao, H. An, and Q. Zhao, "Contour error coupled-control strategy based on line interpolation and curve interpolation," J. of Computers, vol. 8, no. 6, pp. 1512-1519, June 2013.

[13] P. Rodriguez-Ayerbe, D. Dumur, and S. Lavernhe, "Axis control using model predictive control: identification and friction effect reduction," 3rd Int. Conf. on Virtual Machi. Process Tech., p. 8, May 2014.

[14] D. Prévost, S. Lavernhe, C. Lartigue, and D. Dumur, "Feed drive modelling for the simulation of tool path tracking in multi-axis high speed machining," Int. J. of Mechatronics and Manuf. Sys., vol. 4, no. 3-4, pp. 266-284, January 2011. 